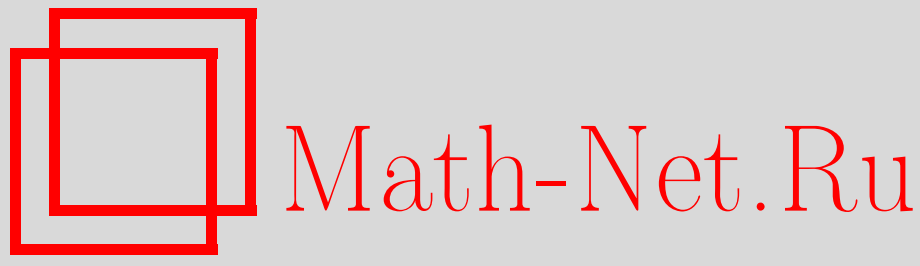

Обцероссийский математический портал

Е. В. Жужома, В. С. Медведев, Лемма о замыкании для кусочно диффеоморфных отображений окружности, Матем. заметки, 2001, том 69, выпуск 2, 310-313

DOI: https://doi.org/10.4213/mzm682

Использование Общероссийского математического портала Math-Net.Ru подразумевает, что вы прочитали и согласны с пользовательским соглашением http://www.mathnet.ru/rus/agreement

Параметры загрузки:

IP : 54.198 .187 .58 
26 апреля 2023 г., 17:48:44

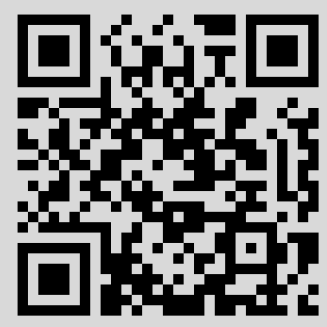




\section{ЛЕММА О ЗАМЫКАНИИ ДЛЯ КУСОЧНО ДИФФЕОМОРФНЫХ ОТОБРАЖЕНИЙ ОКРУЖНОСТИ}

\section{Е.В. ЖУужома, В.С. Медведев}

Введение. Пусть $f$ - кусочно $C^{r}$-диффеоморфное отображение окружности $S^{1}, r \geqslant 1$. В статье доказывается, что если $x \in S^{1}$ - нетривиально рекуррентная точка, то $C^{r}$-малым возмущением ее можно сделать периодической точкой при некотором условии о характере возвращаемости точки $x$ (оно формулируется в терминах вводимых далее “ $t$-разложений”). В качестве следствия мы получаем лемму о замыкании для некоторых потоков с конечным числом неподвижных точек на ориентируемой замкнутой поверхности рода $g \geqslant 2$.

В основном, лемма о замыкании доказывалась для отображений без точек разрьва. В 1939 году Майер [1] доказал $C^{r}$-лемму о замькании для сохраняющих ориентацию диффеоморфизмов $S^{1}$ $(r \geqslant 1)$. Независимо этот результат был передоказан в [2]. В случае $S^{1}$ справедлива даже усиленная лемма о замыкании для блуждаюших цепно рекуррентньх точек [3]. Якобсон [4] доказал $C^{1}$-лемму для $C^{1}$-эндоморфизмов интервала и окружности. В [5] Янг получила $C^{r}$-лемму о замыкании $(2 \leqslant r \leqslant \infty)$ для $C^{r}$-гладких отображений интервала. Пью доказал $C^{1}$-лемму о замькании для диффеоморфизмов и векторных полей на компактных многообразиях [6], [7] (см. также [8]).

Авторы благодарят С. Х. Арансона, В.З. Гринеса и М. И. Малкина за плодотворные обсуждения и Д. В. Аносова за критические замечания, способствовавшие улучшению текста.

Основные определения. Пусть $S^{1} \cong \mathbb{R} / \mathbb{Z}$ - окружность, снабженная положительным направлением, которое индуцируется положительным направлением на $\mathbb{R}$ и универсальным накрытием $\pi: \mathbb{R} \rightarrow S^{1}, \pi(x)=x \bmod 1$. Пусть $\left\{a_{i}\right\}_{i=1}^{k},\left\{b_{i}\right\}_{i=1}^{k}$ - два семейства циклически упорядоченных точек, которые разбивают $S^{1}$ на интервалы $I_{i}=\left(a_{i}, a_{i+1}\right)$ и $J_{i}=\left(b_{i}, b_{i+1}\right)$ соответственно, где $a_{k+1}=a_{1}, b_{k+1}=b_{1}$. Отображение $f: S^{1}-\bigcup_{i=1}^{k} a_{i} \rightarrow S^{1}-\bigcup_{i=1}^{k} b_{i}$ назьвается кусочно $C^{r}$-диффеоморфным,$r \geqslant 0$, если оно взаимно однозначное и ограничение $f$ на каждый интервал $I_{i}$ есть $C^{r}$-гладкий диффеоморфизм на некоторый интервал $J_{\sigma(i)} \cdot\left(C^{0}\right.$-диффеоморфизм является гомеоморфизмом.) Интервалы $I_{i}$ назьвются интервалами диффеоморфности.

Работа выполнена при финансовой поддержке Российского фонда фундаментальных исследований, грант № 99-01-00230, и фонда INTAS, грант № 99-01-00230. 
Введем $C^{r}$-топологию на множестве кусочно диффеоморфных отображений. Обозначим через $\bar{I}_{i}(f)$ замыкание интервала $I_{i}$. Для данного $\varepsilon>0$ будем считать, что кусочно диффеоморфное отображение $g$ принадлежит $\varepsilon$-окрестности $U_{\varepsilon}(f)$ отображения $f$, если существует $\varepsilon$-близкий к тождественному сохраняющий ориентацию $C^{r}$-диффеоморфизм $h: S^{1} \rightarrow S^{1}$ такой, что $h\left(\bar{I}_{i}(f)\right)=$ $\bar{I}_{i}(g)(i=1, \ldots, k)$, и $g$ 。 $h \varepsilon$-близок к $f$ в обычной $C^{r}$-топологии на каждом $\bar{I}_{i}(f)$. Семейство $\varepsilon$-окрестностей $U_{\varepsilon}(f)$ определяет $C^{r}$-топологию. Пространство кусочно диффеоморфных отображений окружности с фиксированным $k$ и наделенное указанной топологией обозначим через $\mathscr{M}^{r+0}(k)$.

Для фиксированного $k$ возьмем множество $S$ символов $J_{1}, \ldots, J_{k}, A_{1}, \ldots, A_{k}$. Пусть $f \in$ $\mathscr{M}^{r+0}(k)$. Для точки $x \in S^{1}$ определим последовательность $i_{f}(x) \in S^{\mathbb{N}}$ следующим образом. Положим $i_{f}(x)=\left(i_{0}(x), \ldots, i_{n}(x), \ldots\right)$, где $i_{n}(x)=J_{i}$, если $f^{n}(x) \in I_{i}$, и $i_{n}(x)=A_{i}$, если $f^{n}(x)=a_{i}$. В последнем случае будем считать $i_{m}(x)=A_{i}$ для всех $m \geqslant n$. Последовательность $i_{f}(x)$ называется маршрутом точки $x$ относительно $f$.

Обозначим $A^{\infty}=\bigcup_{i=0}^{\infty} f^{-i}(A)$ и $B^{\infty}=\bigcup_{i=0}^{\infty} f^{i}(B)$, где $A=\left\{a_{1}, \ldots, a_{k}\right\}$ и $B=\left\{b_{1}, \ldots, b_{k}\right\}$ Если $f^{-i}\left(a_{s}\right)=b_{p}$ для некоторых $1 \leqslant p, s \leqslant k, i \geqslant 1$, то положим $f^{-i-1}\left(a_{s}\right)=f^{-1}\left(b_{p}\right)=\varnothing$. Через $O(x)=\bigcup_{-\infty}^{+\infty} f^{n}(x)$ будем обозначать орбиту точки $x \notin A^{\infty} \cup B^{\infty}$.

Точка $x \notin A^{\infty}$ (соответственно $B^{\infty}$ ) назьвается $\omega$-рекуррентной (соответственно $\alpha$-рекуррентной), если она содержится в своем $\omega$-предельном (соответственно $\alpha$-предельном) множестве. Точка называется рекуррентной, если она $\omega$ - и $\alpha$-рекуррентна. Рекуррентная точка называется нетривиальной, если она не является периодической. Очевидно, орбита рекуррентной точки состоит из рекуррентных точек. Поэтому можно естественным образом ввести понятие рекуррентной орбиты.

Пусть $x \in I_{\nu}=I=\left[a_{\nu}, a_{\nu+1}\right]-$ нетривиально рекуррентная точка кусочно гомеоморфного отображения $f$. Далее будем предполагать, что положительная полуорбита точки $x$ пересекает оба интервала $\left(a_{\nu}, x\right)$ и $\left(x, a_{\nu+1}\right)$. (Это ограничение техническое, поскольку существует точка положительной полуорбиты, для которой данное условие выполняется.) Пусть $q_{1}(r)$ - первая положительная итерация такая, что $f^{q_{1}(r)}(x) \in\left(x, a_{\nu+1}\right)$. Определим по индукции $q_{n}(r)$ как первую итерацию такую, что $f^{q_{n}(r)}(x) \in\left(x, f^{q_{n-1}(r)}(x)\right)$. Аналогично определяются числа $q_{n}(l)$ (например, $q_{1}(l)$ - первая положительная итерация такая, что $\left.f^{q_{1}(l)}(x) \in\left(a_{\nu}, x\right)\right)$.

Рассмотрим блок $B_{n}^{r}=\left\langle i_{0}(x), \ldots, i_{q_{n}(r)}(x)\right\rangle \subset i_{f}(x)$ и определим $r_{n} \in \mathbb{N} \cup\{0\}$ как максимальное число повторений блока $B_{n}^{r}$ в $i_{f}(x)$ после символа $i_{q_{n}(r)}(x)$. Формально, $i_{k}(x)=i_{k+j q_{n}(r)}(x)$ для $0 \leqslant j \leqslant r_{n}, 0 \leqslant k \leqslant q_{n}(r)$. Последовательность $R(x)=\left\{r_{1}(x), \ldots, r_{n}(x), \ldots\right\}$ называется правым $t$-разложсением точки $x$. Поменяв числа $q_{n}(r)$ на $q_{n}(l)$, получим определение левого $t$-разложения точки $x$, которое мы обозначим через $L(x)$.

\section{Леммы о замыкании.}

Теорема 1. Предположим, что кусочно диффеоморфное отображсние $f \in \mathscr{M}^{r+0}(k)$, $r \geqslant 1$, возрастает на каждом интервале диффеоморфности. Пусть $x \in S^{1}$ - нетривиально рекуррентная точка и $L(x)=\left\{l_{i}\right\}_{1}^{\infty}, R(x)=\left\{r_{i}\right\}_{1}^{\infty}$ - ее левое и правое $t$-разложения соответственно. Если

$$
\varlimsup_{n \rightarrow \infty} l_{n} \geqslant 3, \quad \varlimsup_{n \rightarrow \infty} r_{n} \geqslant 3,
$$

то для любой окрестности $U(f)$ отображения $f$ (в $C^{r}$-топологии) существует $\mathfrak{g} \in$ $\mathscr{M}^{r+0}(k) \cap U(f)$, имеющ,е периодическую точку $x$.

Отметим, хоть это и не понадобится в дальнейшем, что утверждаемое в теореме выполняется также и для всех образов точки $x$.

Простейший пример отображения, к которому применима теорема 1 , дает поворот $f: x \mapsto$ $\exp (2 \pi i \mu) x$ с иррациональным $\mu$. Такое преобразование можно считать перекладыванием двух интервалов $\Psi_{1}$ и $\Psi_{2}$ (в выборе которых есть известный произвол). Пусть $y, z-$ концы этих интервалов, $\Lambda_{1}=\left\{f^{j}(y)\right\}_{j=-\infty}^{\infty}, \Lambda_{2}=\left\{f^{j}(z)\right\}_{j=-\infty}^{\infty}, \Lambda=\Lambda_{1} \cup \Lambda_{2}$. Заметим, что для любого $x \in S^{1} \backslash \Lambda$ последовательность $\left\{q_{n}(r)\right\}$ (соответственно $\left.\left\{q_{n}(l)\right\}\right)$ содержит подпоследовательность $\left\{q_{n_{k}}(r)\right\}$ (соответственно $\left\{q_{n_{j}}(l)\right\}$ ), состоящую из знаменателей подходящих дробей числа $\mu$ с четными (соответственно нечетными) номерами. Используя это, можно показать, что при надлежащем выборе 
точек $y, z$ и $x$ последняя имеет требуемые $t$-разложения. При этом можно считать орбиты $\Lambda_{1}$ и $\Lambda_{2}$ дизъюнктньми.

Преобразование поворота - не слишком удачный пример в нашей ситуации, так как для него лемма о замыкании очевидна. Незначительная модификация поворота, однако, дает пример содержательньй. Рассмотрим диффеоморфизм $\varphi: S^{1} \mapsto S^{1}$ (см. [6]) со следующими свойствами:

1) $h \circ \varphi=f \circ h$, где отображение $h: S^{1} \mapsto S^{1}$ непрерьвно и сюръективно;

2) $h\left(\Omega_{1}\right)=y, h\left(\Omega_{2}\right)=z$ для некоторых непустых открытых интервалов $\Omega_{1}$ и $\Omega_{2}$;

3 ) множество блуждающих точек отображения $\varphi$ состоит из $\varphi$-образов интервалов $\Omega_{1}$ и $\Omega_{2}$.

Переопределим $\varphi$ на интервалах $\Omega_{1}$ и $\Omega_{2}$ таким образом, чтобы новое отображение $\psi$ было разрывным. K примеру, пусть $\psi$ афинно отображает $\Omega_{1}$ на $\varphi\left(\Omega_{2}\right)$ и $\Omega_{2}$ на $\varphi\left(\Omega_{1}\right)$. Тогда $t$-разложения точки $x^{\prime}=h^{-1}(x)$ относительно отображения $\psi$ совпадают с соответствующими $t$-разложениями $x$ относительно $f$. Чтобы это увидеть, заметим, что любые два $\psi$-образа точки $x^{\prime}$ принадлежат разным интервалам диффеоморфности отображения $\psi$ тогда и только тогда, когда соответствующие $f$-образы точки $x$ принадлежат разным интервалам $\Psi_{1}$ и $\Psi_{2}$.

Прежде чем формулировать следствия из теоремы 1 , дадим необходимые определения. Пусть $\Delta$ - плоскость Лобачевского, которую мы будем рассматривать в виде единичного диска $|z|<1$ комплексной $z$-плоскости, наделенного метрикой $d s=2|d z| /\left(1-|z|^{2}\right)$. Граница диска $\Delta$ называется абсолютом и обозначается $S_{\infty}$. Любая ориентируемая замкнутая поверхность $M=M_{g}^{2}$ рода $g \geqslant 2$ есть факторпространство $\Delta / \Gamma$, где $\Gamma$ - фуксова группа. Известно, что в качестве фундаментальной области группы $\Gamma$ можно взять геодезический многоугольник $D$ с четным числом сторон, которые попарно отождествляются с помощью образующих $\Gamma_{D} \subset \Gamma$. Обозначим через $N$ сеть, получаемую действием группы $\Gamma$ на границу $\partial D$ многоугольника $D$.

Следуя [10] и [11], припишем каждой стороне $D$ элемент $\Gamma_{D}$ следующим образом: если сторона $s$ отождествляется со стороной $\gamma_{j}(s)$, то $s$ приписывается элемент $\gamma_{j} \in \Gamma_{D}$. Каждому ребру сети $N$ приписьвается элемент, который соответствует стороне $D$, конгруентной данному ребру. Таким образом, любой направленной геодезической $\overline{\mathfrak{g}} \in \Delta$ ставится в соответствие двусторонняя последовательность из образующих, которая описывает последовательность ребер, пересекаемых геодезической. Отметим, что так как каждое ребро $N$ конгруентно двум сторонам многоугольника $D$, то ребра $N$ имеют двойную кодировку, одну “внутреннюю" и одну “внешнюю". Для кодирования геодезической применяется "внутренняя". Полученную последовательность мы будем

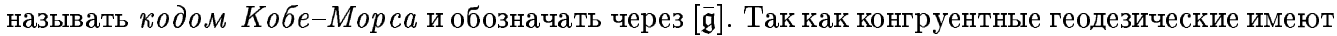
одинаковую (с точностью до сдвига) кодировку, то определяется код Кобе-Морса [a] геодезической $\mathfrak{g} \subset M$ на поверхности.

В работе [12] каждой точке абсолюта $S_{\infty}$ поставлено в соответствие так называемое $f$-разложение, которое представляет собой одностороннюю последовательность образующих $\Gamma_{D} \subset \Gamma$. Объединяя $f$-разложения идеальных концевых точек геодезической $\overline{\mathfrak{g}} \subset \Delta$, мы получим $B S$-разложе ние геодезической $\overline{\mathfrak{g}}$.

Полностью аналогично предыдущему, каждому символу $i_{0} \in[\mathfrak{g}]$ кода Кобе-Морса (или $B S$-разложения) геодезической $g$ и соотвествующей этому символу точке приписываются левое и правое $t$-разложения, которые мы будем обозначать через $l_{n}\left(i_{0}\right)$ и $r_{n}\left(i_{0}\right)(n \geqslant 0)$ соответственно. Для символа $s_{0} B S$-разложения левое и правое $t$-разложения обозначим через $L_{n}\left(s_{0}\right)$ и $R_{n}\left(s_{0}\right)(n \geqslant 0)$ соответственно.

Как следствие теоремы 1 получаем следующие две леммы о замыкании для векторных полей на замкнутых ориентируемых поверхностях $M_{g}^{2}$ рода $g \geqslant 2$ (необходимые определения о траекториях векторных полей см. в [4]).

СледствиЕ 1 . Пусть $X \in \chi^{r}\left(M_{g}^{2}\right)(1 \leqslant r \leqslant \infty, g \geqslant 2)$ - векторное поле, имеющее $E(X)<\infty$ особенностей только седлового типа (т.е. каждый сектор особенности гиперболический). Пусть $l$ - нетривиально рекуррентная траектория поля $X$, проходящая через точку $m \in l$. Предполохим, что существует символ $i_{0} \in[\mathfrak{g}]$ со следующими свойствами:

$$
\varlimsup_{n \rightarrow \infty} r_{n}\left(i_{0}\right) \geqslant 3 E(X)+1, \quad \varlimsup_{n \rightarrow \infty} l_{n}\left(i_{0}\right) \geqslant 3 E(X)+1,
$$

где $[\mathfrak{g}]$ - код Кобе-Морса геодезической $\mathfrak{g}$, соответствующей $l$; тогда найдется векторное 
поле $Y \in \chi^{r}\left(M_{g}^{2}\right)$, произвольно близкое $\kappa X$ (в $C^{r}$-топологии), с периодической траекторией, проходящей через точку $m$.

СледствиЕ 2 . Пусть $X \in \chi^{r}\left(M_{g}^{2}\right)(1 \leqslant r \leqslant \infty, g \geqslant 2)-$ векторное поле, имеющее $E(X)<\infty$ особенностей типа седло, и пусть $l$ - нетривиально рекуррентная траектория поля $X$, проходящая через точку $m \in l$. Предполохим, что ВS-разлохение геодезической $\mathfrak{g}$, соответствующей $l$, имеет символ $s_{0}$ со следующими свойствами:

$$
\varlimsup_{n \rightarrow \infty} R_{n}\left(s_{0}\right) \geqslant 3 E(X)+1, \quad \varlimsup_{n \rightarrow \infty} L_{n}\left(s_{0}\right) \geqslant 3 E(X)+1
$$

тогда найдется векторное поле $Y \in \chi^{r}\left(M_{g}^{2}\right)$, произвольно близкое $\kappa X\left(\right.$ в $C^{r}$-топологии), с периодической траекторией, проходящей через точку $m$.

Гутиеррез [14] доказал $C^{r}$-лемму о замыкании для векторных полей с конечным числом особенностей на торе в предположении, что число вращения векторного поля имеет непостоянный тип (это означает, что непрерывная дробь, в которую раскладывается число вращения, образует неограниченную последовательность). С ледствия 1,2 являются продолжениями результата работы [14] на ориентируемую замкнутую поверхность $M=M_{g}^{2}$ рода $g \geqslant 2$.

\section{СПИСОК ЦИТИРОВАННОЙ ЛИТЕРАТУРЫ}

1. Майер А. Г. // Ученые записки Горьк. госуниверситета. 1939. Т. 12. С. 215-229. 2. Реіхоto M. M. // Topology. 1962. V. 1. P. 101-120; // Topology. 1963. V. 2. P. 179-180. 3. Apaнсон С. Х., ЖКужома Е. В., Медведев В. С. // Матем. заметки. 1997. Т. 61. № 3. С. 323-331. 4. Якобсон М. В. // Матем. сб. 1971. T. 85. С. 183-188. 5. Young L. S. // Invent. Math. 1979. V. 54. P. 179-187. 6. Pugh C. // Amer. J. Math. 1967. V. 89. P. 956-1009. 7. Pugh C. // Amer. J. Math. 1967. V. 89. P. 1010-1021. 8. Pugh C., Robinson C. // Ergodic Theory Dynamical Systems. 1983. V. 3. P. 261-313. 9. Denjoy A. // J. Math. Pure et Appl. 1932. V. 11. P. 333-375. 10. Koebe P. // Sitzung. der Preuss. Akad. der Wissenchaften, 1929. P. 414-457. 11. Morse M. // Amer. J. Math. 1921. V. 43. P. 33-51. 12. Bowen R., Series C. // Publ. IHES. 1979. V. 50. P. 153-170. 13. Aranson S., Belitsky G., Zhuzhoma E. // Transl. of Math. Monographs (AMS). 1996. V. 153. P. 325. 14. Gutierrez C. // Ergodic Theory. Dynamical Systems. 1986. V. 6. P. 45-56.

(Е. В. Жужома) Нижегородский государственный технический университет 